\title{
Analytical approximations for the period of a non-linear pendulum
}

A Beléndez, A Hernández, A Márquez, T Beléndez and C Neipp

Departamento de Física, Ingeniería de Sistemas y Teoría de la Señal.

Universidad de Alicante. Apartado 99. E-03080 Alicante. SPAIN

E-mail: a.belendez@ua.es

Corresponding author: A. Beléndez

Phone: +34-6-5903651

Fax: +34-6-5903464 


\begin{abstract}
This paper deals with the non-linear oscillation of a simple pendulum and presents an approach for solving the non-linear differential equation that governs its movement by using the harmonic balance method. With this technique it is possible to easily obtain analytical approximate formulas for the period of the pendulum. As we shall see, these formulas show excellent agreement with the exact period calculated with the use of elliptical integrals, and they are valid for both small and large amplitudes of oscillation. The most significant feature of the treatment presented is its simplicity because for the level of approximation considered in this paper the required work can be done "by hand".
\end{abstract}

KEY WORDS: Simple pendulum, large-angle period, harmonic balance method 


\section{Introduction}

In physics there are some simple systems for which the equations governing their behaviour are easy to formulate but whose mathematical resolution is complicated. Of all such systems, perhaps the paradigm that is usually considered in physics courses at university level is the simple pendulum. Application of Newton's second law to this physical system gives a differential equation with a non-linear term (the sine of an angle). It is possible to find the integral expression for the period of the pendulum and to express it in terms of elliptic functions. Although it is possible in many cases to replace the non-linear differential equation by a corresponding linear differential equation that approximates the original equation, such linearization is not always feasible. In such cases, the actual non-linear differential equation must be directly dealt with.

There are many approaches for approximating solutions of non-linear oscillatory systems. The most common and most widely studied approximation methods for nonlinear differential equations are the perturbation methods, whereby the solution is analytically expanded in the power series of a small parameter. The simplest of all perturbation methods was developed by Lidstedt and Poincaré and this is the method considered in Marion's book ${ }^{1}$ to approximately solve non-linear differential equations of oscillating systems. Another method of determining the perturbation solution to a non-linear oscillator differential equation was developed by Krylov and Bogoliubov. This method was used by Fulcher and Davis ${ }^{2}$ to calculate the two lowest-order corrections to the period of the simple pendulum. However all these perturbation methods apply to weakly non-linear oscillations only. Through the use of ingenious strategies, different authors ${ }^{3-9}$ have obtained explicit formulas for the period of the pendulum, all of which are accurate for large amplitudes. The non-linear differential equation is linearized by replacing $\sin \theta$ by $f\left(\theta_{0}\right) \theta$ instead of by $\theta$, where $f\left(\theta_{0}\right)$ is an unknown function of the amplitude of oscillation to be determined. The approximate period is then a function of $f\left(\theta_{0}\right)$. These authors suggest different functional forms for $f\left(\theta_{0}\right)$ with a set of unknown parameters that are evaluated by comparing the power-series expansions of the approximate and exact periods. However all these authors do a heuristic derivation of their formulas. 
The harmonic balance method ${ }^{10,11}$ is another procedure for determining analytical approximations to the periodic solutions of differential equations by using a truncated Fourier series representation. An important advantage of this method is that it can be applied to non-linear oscillatory problems where the non-linear terms are not small, i. e., no perturbation parameter needs to appear. ${ }^{10}$ The perturbation methods are, in principle, for solving problems with a small parameter. In that case, the solution is analytically expanded in power series of the parameter. However, there exist many non linear problems in which parameters are not small and other methods such as that of the harmonic balance are able to provide analytical approximations valid for large values of the amplitude. With the aid of this method it is possible to produce analytical approximations to the period and periodic solution of non-linear oscillators. These approximated solutions are valid even for rather large amplitudes of oscillation. There exists an ample literature on the harmonic balance method and a wide range of analysis and applications of this method to a great variety of differential equations, which can be found in Mickens' book ${ }^{10}$, where the details of the general procedures for calculating solutions by means of this method are specified. This book also illustrates the use of harmonic balance by applying it to a number of important non-linear differential equations and the application of this method to more complex oscillatory systems ${ }^{11,12}$ has also been successfully done. An important aspect to take into account is that for conservative systems in which the non-linear differential equation has the expression $\mathrm{d}^{2} u / \mathrm{d} t^{2}+u=\varepsilon F(u)$, where $F(-u)=-F(u)$, for small values of the parameter $\varepsilon$, the harmonic balance first approximation solution agrees well with the determined by means of the Lindstedt-Poincaré technique ${ }^{10}$. Moreover, for these systems, the harmonic balance method provides good analytical approximations to the periodic solutions even for large values of $\varepsilon^{10}$.

In this paper we obtain analytical approximate formulas for the period of a simple pendulum by using the harmonic balance method. Firstly the non-linear differential equation that governs the oscillatory movement of the simple pendulum is considered. Next the trigonometrical function $\sin \theta$ that appears in this non-linear differential equation is replaced by its power series expansion, and the resulting equation is approximately solved by means of the harmonic balance method. To do this the power series expansion of $\sin \theta$ is truncated by considering different terms. When we apply the 
harmonic balance method we only consider the first approximation to the periodic solution to the non-linear differential equation, because for this approximation the required work can usually be done "by hand". We can also see that this method is capable of producing a first analytical approximation to the solution to the simple pendulum, and its accuracy is very good in a wide range of amplitudes of oscillation (as high as $117^{\circ}$ ). Finally, we will see that one of the most interesting features of this approximation is its simplicity.

\section{Exact solution}

The differential equation modeling the free, undamped simple pendulum is

$$
\frac{\mathrm{d}^{2} \theta}{\mathrm{d} t^{2}}+\frac{g}{l} \sin \theta=0
$$

where $\theta$ is the angular displacement, $l$ is the length of the pendulum, $t$ is the time and $g$ is the acceleration due to gravity. The oscillations of the pendulum are subjected to the initial conditions

$$
\theta(0)=\theta_{0} \quad\left(\frac{\mathrm{d} \theta}{\mathrm{d} t}\right)_{t=0}=0
$$

where $\theta_{0}$ is the amplitude of oscillation. The system oscillates between symmetric limits $\left[-\theta_{0},+\theta_{0}\right]$. The periodic solution $\theta(t)$ of equation (1) and the angular frequency $\omega$ (also with the period $T=2 \pi / \omega$ ) depend on the amplitude $\theta_{0}$.

Equation (1), although straightforward in appearance, is in fact rather difficult to solve because of the non-linearity of the trigonometric function $\sin \theta$. There are no analytical solutions for the above differential equation. In fact, the solution is expressed in terms of elliptic integrals ${ }^{8}$. Hence, equation (1) is either solved numerically or various approximations are used. In the most simple of these approximations we consider that the angle $\theta$ is small, and then the function $\sin \theta$ can be approximated by $\theta$. Then the nonlinear differential equation (1) becomes a linear differential equation that can easily be solved, and the period $T_{0}$ of the oscillation is given by 


$$
T_{0}=2 \pi \sqrt{\frac{l}{g}}
$$

The period for this case is independent of the amplitude $\theta_{0}$ of oscillations and it is only a function of the length $l$ of the pendulum and the acceleration of gravity $g$.

The exact value of the period of oscillations is given by the equation ${ }^{1}$

$$
T_{\mathrm{ex}}=T_{0} \frac{2}{\pi} K(k)
$$

where $k=\sin ^{2}\left(\theta_{0} / 2\right)$ and $K(k)$ is the complete elliptic integral of the first kind. Its values have been tabulated for various values of $k$. The power-series expansion of the equation (4) is $^{2}$

$$
T_{\mathrm{ex}} \approx T_{0}\left(1+\frac{1}{4} k^{2}+\frac{9}{64} k^{4}+\frac{25}{256} k^{6}+\ldots\right)
$$

Using the power series expansion of $k=\sin ^{2}\left(\theta_{0} / 2\right)$, we may write another series for the exact period

$$
T_{\mathrm{ex}} \approx T_{0}\left(1+\frac{1}{16} \theta_{0}^{2}+\frac{11}{3072} \theta_{0}^{4}+\frac{173}{737280} \theta_{0}^{6}+\frac{22931}{1321205760} \theta_{0}^{8}+\ldots\right)
$$

Equations (4) and (6) are the forms that we shall use to compare with the harmonic balance method calculation in the following sections.

\section{Formulation and approximate solution method}

As we mentioned previously, to obtain an analytical approximate solution for equation (1) using the harmonic balance method, first we expand $\sin \theta$ in its Maclaurin series expansion

$$
\sin \theta=\sum_{n=0}^{\infty} \frac{(-1)^{n}}{(2 n+1) !} \theta^{2 n+1}
$$

and substituting equation (7) into equation (1) gives

$$
\frac{\mathrm{d}^{2} \theta}{\mathrm{d} t^{2}}+\omega_{0}^{2} \sum_{n=0}^{\infty} \frac{(-1)^{n}}{(2 n+1) !} \theta^{2 n+1}=0
$$


where $\omega_{0}$ is a known constant parameter that is given by the expression

$$
\omega_{0}=\sqrt{\frac{g}{l}}
$$

The harmonic balance method is very simple and allows us to obtain high quality approximate solutions if the exact solution (which is unknown) resembles a harmonic function (sine or cosine). If the unknown solution is periodic, this solution can be expressed as a Fouries series ${ }^{10}$

$$
\theta(t)=\sum_{n=1}^{\infty}\left[A_{n} \sin (n \omega t)+B_{n} \cos (n \omega t)\right]
$$

where $A_{n}$ and $B_{n}$ are the Fourier coefficients.

Based on equation (1), the periodic solution $\theta(t)$ for the simple pendulum has a Fourier series expansion

$$
\theta(t)=\sum_{n=0}^{\infty} A_{2 n+1} \cos [(2 n+1) \omega t]
$$

which contains only odd multiples of $\omega t .^{10,11}$

The purpose of the harmonic balance method is to approximate the periodic solution of equation (11) by a trigonometric polynomial

$$
\theta(t)=\sum_{n=0}^{N} A_{2 n+1} \cos [(2 n+1) \omega t]
$$

and determine both the coefficients $A_{2 n+1}$ and the angular frequency $\omega$. All of these quantities are to be expressed finally in terms of the initial conditions -in this problem they will be a function of the amplitude $\theta_{0^{-}}$.

Following the lowest order harmonic balance method, a reasonable and simple initial approximation satisfying initial conditions in equation (2) can be taken as

$$
\theta(t)=\theta_{0} \cos \omega t
$$

where we have taken into account the initial condition $\theta(0)=\theta_{0}$ and $\theta(t)$ is a periodic function of $t$, of period $T=2 \pi / \omega$. Equation (13) is the first analytical approximate periodic solution of equation (8), known as the "first approximation". 
Applying the harmonic balance method we need to obtain an approximate solution of equation (8) with the functional form of equation (13). It is necessary to obtain the optimal expression of the frequency $\omega$ (or the period $T$ ) which makes the approximate periodic solution proposed a good representation of the unknown exact periodic solution. With this method, the value of $\omega$ determined is such that the approximate solution proposed (13) satisfies equation (8) (or equation (1)) in its highest harmonics, which are usually those of the lowest order ${ }^{10}$.

Substituting equation (13) into equation (8) gives

$$
-\omega^{2} \theta_{0} \cos \omega t+\omega_{0}^{2} \sum_{n=0}^{\infty} \frac{(-1)^{n}}{(2 n+1) !} \theta_{0}^{2 n+1} \cos ^{2 n+1} \omega t=0
$$

The formula that allows us to obtain the odd power of the cosine is

$$
\cos ^{2 n+1} \omega t=\frac{1}{2^{2 n}}\left\{\cos (2 n+1) \omega t+\left(\begin{array}{c}
2 n+1 \\
1
\end{array}\right) \cos (2 n-1) \omega t+\ldots+\left(\begin{array}{c}
2 n+1 \\
n
\end{array}\right) \cos \omega t\right\}
$$

Substituting equation (15) into equation (14) gives

$$
-\omega^{2} \theta_{0} \cos \omega t+\omega_{0}^{2} \sum_{n=0}^{\infty} \frac{(-1)^{n}}{(2 n+1) !} \theta_{0}^{2 n+1} \frac{1}{2^{2 n}}\left(\begin{array}{c}
2 n+1 \\
n
\end{array}\right) \cos \omega t+H O H=0
$$

where $H O H$ are high order harmonics, in other words, proportional to $\cos [(2 n+1) \omega t]$ for $n \geq 1$. Equation (16) can be rewritten as follows

$$
\left[-\omega^{2}+\omega_{0}^{2} \sum_{n=0}^{\infty} \frac{(-1)^{n}}{2^{2 n} n !(n+1) !} \theta_{0}^{2 n}\right] \cos \omega t+H O H=0
$$

For the lowest order harmonic to be equal to zero, it is necessary to set the coefficient of $\cos \omega t$ equal to zero in equation (17), and for $\omega$ we obtain the following expression

$$
\omega=\omega_{0}\left[\sum_{n=0}^{\infty} \frac{(-1)^{n}}{2^{2 n} n !(n+1) !} \theta_{0}^{2 n}\right]^{1 / 2}
$$

which gives us the approximate frequency $\omega$ as a function of $\theta_{0}$.

Taking into account the relation $T=2 \pi / \omega$ and equations (3) and (9), the approximate period $T_{\text {app }}$ of oscillations can be obtained as follows 


$$
T_{\text {app }}=T_{0}\left[\sum_{n=0}^{\infty} \frac{(-1)^{n}}{2^{2 n} n !(n+1) !} \theta_{0}^{2 n}\right]^{-1 / 2}
$$

which allows us to obtain $T_{\text {app }}$ as a function of the amplitude $\theta_{0}$ of oscillation.

We shall now study various specific cases in which a different number of terms is considered in the power-series expansion of equation (7). Thus, if we take $N$ terms of this series the values of the different approximate periods $T_{\text {appN }}$ may be calculated using the equation

$$
T_{\text {appN }}=T_{0}\left[\sum_{n=0}^{N} \frac{(-1)^{n}}{2^{2 n} n !(n+1) !} \theta_{0}^{2 n}\right]^{-1 / 2}
$$

\section{Specific case $N=0$}

This situation implies taking only $N=0$ in equation (7) and then $\sin \theta \approx \theta$. Therefore, from equation (20) the approximate period is $T_{\text {app } 0}=T_{0}$ (equation (3)).

\section{Specific case $N=1$}

Now the approximation considered in equation (7) is

$$
\sin \theta \approx \theta-\frac{1}{6} \theta^{3}
$$

and equation (20) becomes

$$
T_{\text {app } 1}=T_{0}\left(1-\frac{1}{8} \theta_{0}^{2}\right)^{-1 / 2}
$$

which is only applicable for $\theta_{0}<\sqrt{8} \mathrm{rad}=2.828 \mathrm{rad}\left(\approx 162^{\circ}\right)$.

For small angles it is possible to do the power-series expansion of the right-hand side of equation (22). Doing this gives the result

$$
T_{\text {app } 1} \approx T_{0}\left(1+\frac{1}{16} \theta_{0}^{2}+\frac{18}{3072} \theta_{0}^{4}+\ldots\right)
$$


It is important to point out that in equation (23) the first two terms are the same as the first two terms of the equation obtained in the power-series expansion of the exact period (equation (6)), whereas, in equation (23) the third term is 18/3072 as compared with $11 / 3072$ in the series expansion of the exact period.

\section{Specific case $N=2$}

Now the approximation considered in equation (7) is

$$
\sin \theta \approx \theta-\frac{1}{6} \theta^{3}+\frac{1}{120} \theta^{5}
$$

and equation (20) becomes

$$
T_{\text {app2 }}=T_{0}\left(1-\frac{1}{8} \theta_{0}^{2}+\frac{1}{192} \theta_{0}^{4}\right)^{-1 / 2}
$$

doing the power-series expansion of the right-hand side of equation (25) the following equation can be obtained

$$
T_{\mathrm{app} 2} \approx T\left(1+\frac{1}{16} \theta_{0}^{2}+\frac{10}{3072} \theta_{0}^{4}+\frac{90}{737280} \theta_{0}^{6}+\ldots\right)
$$

In this equation, as in the case $N=1$, the first two terms are the same as the first two terms obtained from the expansion of $T$ of the elliptic integral (equation (6)), whereas the third term of the expansion of the exact period is 11/3072 compared with $10 / 3072$ obtained in our study, that is, the relative error in this term is $10 \%$. In the fourth term a coefficient of $90 / 737280$ is obtained whereas the exact value is $173 / 737280$.

\section{Specific case $N=3$}

The approximation considered in equation (7) is now:

$$
\sin \theta \approx \theta-\frac{1}{6} \theta^{3}+\frac{1}{120} \theta^{5}-\frac{1}{5040} \theta^{7}
$$

and from equation (17) the following expression can be obtained

$$
T_{\mathrm{app} 3}=T_{0}\left(1-\frac{1}{8} \theta_{0}^{2}+\frac{1}{192} \theta_{0}^{4}-\frac{1}{9216} \theta_{0}^{6}\right)^{-1 / 2}
$$

The power-series expansion of the right-hand side of equation (28) gives 


$$
T_{\mathrm{app} 3} \approx T_{0}\left(1+\frac{1}{16} \theta_{0}^{2}+\frac{10}{3072} \theta_{0}^{4}+\frac{130}{737280} \theta_{0}^{6}+\ldots\right)
$$

As in the cases $N=1$ and $N=2$, the first two terms are the same as the first two terms obtained from expansion of the exact period $T$ of the elliptic integral (equation (6)), whereas the third term of the expansion of the exact period is $11 / 3072$ compared with 10/3072 obtained in this study. However, compared with the value obtained with $n$ $=2$, in this case we obtain a coefficient of the fourth term of 130/737280 whereas the exact value is $173 / 737280$.

\section{Specific case $N=4$}

The approximation considered in equation (7) is now:

$$
\sin \theta \approx \theta-\frac{1}{6} \theta^{3}+\frac{1}{120} \theta^{5}-\frac{1}{5040} \theta^{7}+\frac{1}{362880} \theta^{9}
$$

and equation (20) may be written

$$
T_{\text {app } 4}=T_{0}\left(1-\frac{1}{8} \theta_{0}^{2}+\frac{1}{192} \theta_{0}^{4}-\frac{1}{9216} \theta_{0}^{6}+\frac{1}{737280} \theta_{0}^{8}\right)^{-1 / 2}
$$

As in the previous cases, the power-series expansion of equation (31) is done and this gives

$$
T_{\mathrm{app} 4} \approx T_{0}\left(1+\frac{1}{16} \theta_{0}^{2}+\frac{10}{3072} \theta_{0}^{4}+\frac{130}{737280} \theta_{0}^{6}+\frac{13384}{1321205760} \theta_{0}^{8}+\ldots\right)
$$

which coincides up to the fourth term with the series expansion of equation (29).

\section{General case $N=\infty$}

In the most general case no approximation of the series expansion of equation (7) is made, and this amounts to solving the differential equation (1) or its equivalent equation (8). In order to obtain the approximate solution to equation (8) it is only necessary to take into consideration that the series-expansion of Bessel's function of the first kind and order is

$$
J_{1}\left(\theta_{0}\right)=\sum_{n=0}^{\infty} \frac{(-1)^{n}}{2^{2 n+1} n !(n+1) !} \theta_{0}^{2 n+1}
$$


Comparing equation (20) and equation (33) it can be seen that the approximate equation for the period (19) may also be written as

$$
T_{\text {app }}=T_{0} \sqrt{\frac{\theta_{0}}{2 J_{1}\left(\theta_{0}\right)}}
$$

In this case, we do not obtain an analytical function for the period and in principle evaluation of equation (34) poses the same difficulties as calculation of the exact period from elliptic functions. However, we have included it since it represents the best approximation bearing in mind the first approximation in the harmonic balance method. In the following section it will be seen that for errors less than $1 \%$ when $N=3$ practically the same results are obtained as in this general case.

The power-series expansion of equation (34) is

$$
T_{\mathrm{app}} \approx T_{0}\left(1+\frac{1}{16} \theta_{0}^{2}+\frac{10}{3072} \theta_{0}^{4}+\frac{130}{737280} \theta_{0}^{6}+\frac{13384}{1321205760} \theta_{0}^{8}+\ldots\right)
$$

which coincides up to the fifth term with the series expansion of equation (32). However, both equations differ in the sixth term of the series (power of $\theta^{10}$ ).

\section{Comparison between exact and approximate solutions}

In this section, we illustrate the applicability, accuracy and effectiveness of the approach by comparing the analytical approximate period with the exact solution.

The values of four expressions $T_{\mathrm{ex}} / T_{0}$ (equation (6)), $T_{\mathrm{appl}} / T_{0}$ (equation (22)), $T_{\text {app } 2} / T_{0}$ (equation (25)), $T_{\text {app } 3} / T_{0}$ (equation (28)), $T_{\text {app } 4} / T_{0}$ (equation (31)) and $T_{\text {app }} / T_{0}$ (equation (34)) for different values of the amplitude $\theta_{0}$ are presented in Table 1 . This table illustrates very good agreement between approximate periods and the exact period for a wide range of values of oscillation amplitude. Table 1 also shows that the analytical approximate period $T_{\text {app3 }}$ gives better accuracy than the other approximations and similar accuracy to the non-analytical approximation period $T_{\text {app. }}$. This means that for the first approximation of the harmonic balance method, it is sufficient to consider up to $N=3$ in equation (7), since for $N \geq 4$ the results cannot be improved. In order to achieve an improvement it is necessary to construct the second approximation to the 
periodic solution (taking into consideration two terms in equation (12), that is, $N=2$ ). This is shown in the appendix, since as can be seen higher approximations do not add anything new from the qualitative point of view. The relative error between the approximate values and the exact value is less than $1 \%$ for $\theta_{0}<23^{\circ}$ if we consider the approximation $N=0, \theta_{0}<78^{\circ}$ for $N=1, \theta_{0}<107^{\circ}$ for $N=2, \theta_{0}<117^{\circ}$ for $N=3, \theta_{0}<$ $117^{\circ}$ for $N=4$ and $\theta_{0}<117^{\circ}$ for $N=\infty$.

In figure 1, we also present a comparison of approximations $T_{\mathrm{app} 2}, T_{\mathrm{app} 3}, T_{\mathrm{app} 4}$ and

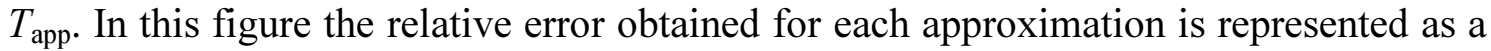
function of the amplitude in a range of values from $80^{\circ}$ to $150^{\circ} . T_{\text {app } 1}$ is not presented, since a relative error higher than $1 \%$ is obtained in this range of values. As can be seen in the figure, the best approximation is the one given by $T_{\text {app3 }}$. The relative error of this approximation and that of $T_{\text {app } 4}$ and $T_{\text {app }}$ is kept even lower than 5\% for amplitudes as

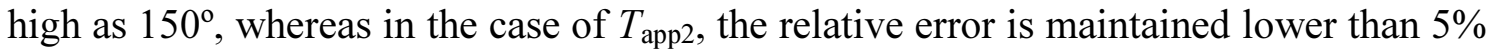
when the amplitude is smaller than $138^{\circ}$.

Taking into account Table 1 and figure 1 it may be concluded that if the approximate solution considered is the cosenoidal function of equation (13), that is, $\theta(s)=\theta_{0} \cos \omega t$, the maximum value of the amplitude of oscillation, for which the relative error of the approximate period compared with the exact period is less than $1 \%$, is $\theta_{0}=117^{\circ}$, and it is impossible to obtain relative errors less than $1 \%$ for values greater than $\theta_{0}=117^{\circ}$. In order to maintain the relative error at less than $1 \%$ with greater amplitudes, it is necessary to consider a solution like that in equation (12) but including a term corresponding to the higher harmonic, that is $\cos 3 \omega t$. Since we wish to calculate manually the analytical approximations to the exact solutions, the number of harmonics have to be small. The main reason is the complexity of the algebra involved. However, this is not a major restriction because, as we have shown, equations obtained in the previous section are capable of providing excellent analytical approximate representations of the exact period for small as well as large amplitudes of oscillation. In the appendix the solutions obtained when the second approximation in the harmonic balance method is considered are presented. 


\section{Conclusions}

The formula for the period of the simple pendulum for small angles commonly given in introductory textbooks is only the limiting case of a much wider range of real world behavior. We have shown that it is easy to extend the theory to the case of large amplitudes and to obtain analytical approximate expressions for the period. In particular, various analytical approximation formulas for the period of a simple pendulum have been obtained by using the harmonic balance method. As can be seen the analysis of the non-linear pendulum by means the application of this technique using the first analytical approximation is very simple in its principle, and is very easy to implement. Excellent agreement of the analytical approximate period with the exact period has been demonstrated not only for small but also for large amplitudes (as high as $117^{\circ}$ ). The analysis considered in this paper demonstrates that, unlike perturbation approximations, the present analytical approximate periods apply well to small as well as large values of amplitude of oscillation. Finally, in this paper not only did we reveal some interesting aspects of the study of non-linear oscillating systems -such as the simple pendulum- or procedures for determining analytical approximations to the periodic solutions of non-linear differential equations, but we also presented an application of one of these procedures -the method of harmonic balance- to easily obtain analytical approximations for the period which have some pedagogical value to both graduate as well as undergraduate students.

\section{Appendix}

This appendix presents the details of how to construct the second approximation to the periodic solutions to different approximate nonlinear differential equations for the simple pendulum. In order to obtain the second approximation we use the approach developed by Lim et $\mathrm{al}^{11,12}$. They simplify the harmonic balance method by imposing linearization of the governing equation in order to avoid numerically solving sets of equations with very complex nonlinearities and accurate analytical approximate periods 
can be obtained easily. This linearization is performed prior to proceeding with harmonic balancing.

We only consider in detail the specific case $N=2$. For this case Eq. (8) can be written as follows:

$$
\frac{\mathrm{d}^{2} \theta}{\mathrm{d} t^{2}}+\omega_{0}^{2}\left(\theta+a \theta^{3}+b \theta^{5}\right)=0
$$

where $a=-1 / 6$ and $b=1 / 120$. By using Eq. (12), the second approximation form for the periodic solution to Eq. (A1) is:

$$
\theta(t)=A_{1} \cos \omega t+A_{2} \cos 3 \omega t
$$

Since $\theta(0)=\theta_{0}$, it follows that:

$$
\theta_{0}=A_{1}+A_{2}
$$

and then:

$$
A_{1}=\theta_{0}-A_{2}
$$

Substituting Eq. (A4) into Eq. (A2) we obtain:

$$
\theta(t)=\theta_{0} \cos \omega t+A_{2}(\cos 3 \omega t-\cos \omega t)
$$

Eq. (A5) can be rewritten in the form:

$$
\theta(t)=u(t)+v(t)
$$

Where $u(t)$ is the main part satisfying initial conditions in Eq. (2)

$$
u(0)=\theta_{0} \quad\left(\frac{\mathrm{d} u}{\mathrm{~d} t}\right)_{t=0}=0
$$

and takes the form of Eq. (13):

$$
u(t)=\theta_{0} \cos \omega t
$$

and $v(t)$ is a correction term

$$
v(t)=A_{2}(\cos 3 \omega t-\cos \omega t)
$$

It is easy to verify that:

$$
v(0)=0 \quad\left(\frac{\mathrm{d} v}{\mathrm{~d} t}\right)_{t=0}=0
$$


Substituting Eq. (A6) into Eq. (A1) and making linearization of the resulting equation with respect to the correction $v(\mathrm{t})$, we obtain:

$$
\frac{\mathrm{d}^{2} u}{\mathrm{~d} t^{2}}+\frac{\mathrm{d}^{2} v}{\mathrm{~d} t^{2}}+\omega_{0}^{2}\left[u+v+a u^{3}+b u^{5}+3 a u^{2} v+5 b u^{4} v\right]=0
$$

where in the linearization process the terms containing the nth powers of $v$ with $n \geq 2$, have been removed. Solving the resulting Eq. (A11) by the method of harmonic balance, it could be obtained the approximate period and the periodic solution. Substituting Eqs. (A8) and (A9) into Eq. (A11), and taking into account the trigonometric relation

$$
\cos 3 \omega t=4 \cos ^{3} \omega t-3 \cos \omega t
$$

we obtain, after some algebraic manipulation, that:

$$
\begin{aligned}
& \left.-\left[\left(\theta_{0}-28 A_{2}\right) \omega^{2}+\left(\theta_{0}-4 A_{2}\right) \omega_{0}^{2}\right)\right] \cos \omega t-\left[36 A_{2} \omega^{2}-\left(a \theta_{0}^{3}+4 A_{2}-12 a \theta_{0}^{2} A_{2}\right) \omega_{0}^{2}\right] \cos ^{3} \omega t+ \\
& +\left[\left(-12 a \theta_{0}^{2} A_{2}+b \theta_{0}^{5}-20 b \theta_{0}^{4} A_{2}\right) \omega_{0}^{2}\right] \cos ^{5} \omega t+\left[20 b \theta_{0}^{4} A_{2} \omega_{0}^{2}\right] \cos ^{7} \omega t=0
\end{aligned}
$$

and taking into account Eq. (15) for $\cos ^{3} \omega t, \cos ^{5} \omega t$ and $\cos ^{7} \omega t$, we obtain:

$$
\begin{aligned}
& \frac{1}{16}\left[\left(12 a \theta_{0}^{3}-24 a \theta_{0}^{2} A_{2}+10 b \theta_{0}^{5}-25 b \theta_{0}^{4} A_{2}\right) \omega_{0}^{2}+16\left(\theta_{0}-A_{2}\right)\left(\omega_{0}^{2}-\omega^{2}\right)\right] \cos \omega t+ \\
& +\frac{1}{16}\left[\left(4 a+5 b \theta_{0}^{2}\right) \theta_{0}^{3} \omega_{0}^{2}+\left(144 \omega^{2}+\left(16+12 a \theta_{0}^{2}+5 b_{0}^{4}\right) \omega_{0}^{2}\right) A_{2}\right] \cos 3 \omega t+H O H=0
\end{aligned}
$$

where $\mathrm{HOH}$ are high order harmonics, in other words, proportional to $\cos [(2 n+1) \omega t]$ for $n \geq 2$. Equating the coefficients of $\cos \omega t$ and $\cos 3 \omega t$ to zero gives the two equations:

$$
\left(12 a \theta_{0}^{3}-24 a \theta_{0}^{2} A_{2}+10 b \theta_{0}^{5}-25 b \theta_{0}^{4} A_{2}\right) \omega_{0}^{2}+16\left(\theta_{0}-A_{2}\right)\left(\omega_{0}^{2}-\omega^{2}\right)=0
$$

$$
\left(4 a+5 b \theta_{0}^{2}\right) \theta_{0}^{3} \omega_{0}^{2}-\left(144 \omega^{2}-\left(16+12 a \theta_{0}^{2}+5 b_{0}^{4}\right) \omega_{0}^{2}\right) A_{2}=0
$$

Eliminating $A_{2}$ from Eqs. (A15) and (A16), we get

$$
A \omega^{4}+B \omega^{2}+C=0
$$

which can be solved for $\omega$ as a function of $\theta_{0}$. In Eq. (A17) coefficients $A, B$ and $C$ are given by

$$
A=2304 \theta_{0}
$$




$$
\begin{gathered}
B=\left(-2560 \theta_{0}-1984 a \theta_{0}^{3}-1600 b \theta_{0}^{5}\right) \omega_{0}^{2} \\
C=\left(256 \theta_{0}+488 a \theta_{0}^{3}+240 a^{2} \theta_{0}^{5}+320 b \theta_{0}^{5}+400 a b \theta_{0}^{7}+175 b^{2} \theta_{0}^{9}\right) \omega_{0}^{4}
\end{gathered}
$$

Eq. (A17) can be solved for the second analytical approximate frecuency $\omega$ as a function of the amplitude of the oscillations, $\theta_{0}$ :

$$
\omega\left(\theta_{0}\right)=\frac{\omega_{0}}{12} \sqrt{80+62 a \theta_{0}^{2}+50 b \theta_{0}^{4}+\sqrt{\Delta\left(\theta_{0}\right)}}
$$

where:

$$
\Delta\left(\theta_{0}\right)=4096+5888 a \theta_{0}^{2}+1684 a^{2} \theta_{0}^{4}+5120 b \theta_{0}^{4}+2600 a b \theta_{0}^{6}+925 b^{2} \theta_{0}^{8}
$$

The sign "+" before $\sqrt{\Delta\left(\theta_{0}\right)}$ in Eq. (A21) has been determined by the condition that the ratio $\omega$ approaches $\omega_{0}$ for $\theta_{0}$ approaching 0 . Furthemore, $A_{2}$ in Eq. (A9) can be obtained by using either Eq. (A15) or Eq. (A16): i.e.,

$$
A_{2}\left(\theta_{0}\right)=\frac{16 \theta_{0}+12 a \theta_{0}^{3}+10 b \theta_{0}^{5}-16 \theta_{0}\left[\omega\left(\theta_{0}\right) / \omega_{0}\right]^{2}}{16+24 a \theta_{0}^{2}+25 b \theta_{0}^{4}-16\left[\omega\left(\theta_{0}\right) / \omega_{0}\right]^{2}}
$$

The corresponding period for the second approximation as a function of $\theta_{0}$ is given by:

$$
T_{\text {app2 }}=T_{0} \frac{12}{\sqrt{80+62 a \theta_{0}^{2}+50 b \theta_{0}^{4}+\sqrt{\Delta\left(\theta_{0}\right)}}}
$$

where $\Delta\left(\theta_{0}\right)$ is given by Eq. (A22) and $a=-1 / 6$ and $b=1 / 120$. The relative error between the approximate value and the exact value (Eq. (4)) is less than $1 \%$ for $\theta_{0}<$ $122^{\circ}$. Furthermore, the second analytical approximate periodic solution is given by:

$$
\theta(t)=\left[\theta_{0}-A_{2}\right] \cos \omega t-A_{2} \cos 3 \omega t
$$

By expanding in power-series the right-hand side of equation (A24) and taking into account that $a=-1 / 6$ and $b=1 / 120$, the following equation can be obtained

$$
T_{\text {app } 2} \approx T_{0}\left(1+\frac{1}{16} \theta_{0}^{2}+\frac{11}{3072} \theta_{0}^{4}+\frac{133.75}{737280} \theta_{0}^{6}+\frac{9356.67}{1321205760} \theta_{0}^{8}+\ldots\right)
$$

In this equation, the first three terms are the same as the first three terms obtained from the expansion of the exact period $T$ (Eq (6)), whereas the fourth term of the expansion of the exact period is $173 / 737280$ compared with $133.75 / 737280$ obtained in this study, that is, the relative error in this term is $23 \%$. 
By doing the same procedure in the case $N=3$, it can be obtained a new period,

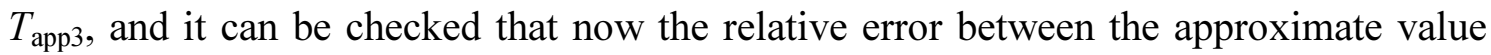
and the exact value is less than $1 \%$ for $\theta_{0}<151^{\circ}$. By expanding in power-series the analytical period obtained by using the second approximation for $N=3$, it is obtained:

$$
T_{\mathrm{app} 3} \approx T_{0}\left(1+\frac{1}{16} \theta_{0}^{2}+\frac{11}{3072} \theta_{0}^{4}+\frac{173.75}{737280} \theta_{0}^{6}+\frac{24140.7}{1321205760} \theta_{0}^{8}+\ldots\right)
$$

In this equation, the first three terms are the same as the first three terms obtained from the expansion of the exact period $T$ (Eq. (6)), whereas the fourth term of the expansion of the exact period is $173 / 737280$ compared with $173.75 / 737280$ obtained in our study, that is, the relative error in this term is $0.4 \%$. In the fifth term a coefficient of $24140.7 / 1321205760$ is obtained whereas the exact value is $22931 / 1321205760$. The relative error for this coefficient is $5 \%$.

Next, by doing the same procedure in the case $N=4$, it can be obtained a new period, $T_{\text {app } 4}$, and it can be checked that now the relative error between the approximated value and the exact value is less than $1 \%$ for $\theta_{0}<162^{\circ}$. Doing the power-series expansion of analytical period obtained by using the second approximation for $N=4$, it is obtained: $T_{\text {app } 4} \approx T_{0}\left(1+\frac{1}{16} \theta_{0}^{2}+\frac{11}{3072} \theta_{0}^{4}+\frac{173.75}{737280} \theta_{0}^{6}+\frac{23244.7}{1321205760} \theta_{0}^{8}+\ldots\right)(\mathrm{A} 2$

In this equation, the first three terms are the same as the first three terms obtained from the expansion of the exact period $T$ (Eq. (6)), whereas the fourth term of the expansion of the exact period is $173 / 737280$ compared with $173.75 / 737280$ obtained in our study, that is, the relative error in this term is $0.4 \%$. In the fifth term a coefficient of $23244.7 / 1321205760$ is obtained whereas the exact value is $22931 / 1321205760$. The relative error for this coefficient is $1.4 \%$.

Finally, it is necessary to point out that only the second approximation for $N=2$, 3 and 4 has been obtained, since these are the ones for which the third term of the power series expansion of the obtained periods with the first approximation (Eqs. (26), (29) and (32)) is 10/3072 against a 11/3072 of the third term of the series expansion of the exact period (Eq. (6)). In the case $N=1$, the second approximation is also easily obtained, since it is only needed to make $b=0$ in Eqs. (A22) and (A.24). The obtained result is: 


$$
T_{\mathrm{app} 1}=T_{0} \frac{12}{\sqrt{80+62 a \theta_{0}^{2}+2 \sqrt{1024+1472 a \theta_{0}^{2}+421 a^{2} \theta_{0}^{4}}}}
$$

Doing the power-series expansion of Eq. (A29) taking into account that $a=-1 / 6$, it is obtained:

$$
T_{\mathrm{app} 1} \approx T_{0}\left(1+\frac{1}{16} \theta_{0}^{2}+\frac{19}{3072} \theta_{0}^{4}+\frac{419}{589824} \theta_{0}^{6}+\frac{10042}{113246208} \theta_{0}^{8}+\ldots\right)
$$

It can be seen how for the case $N=1$, both for the second approximation and for the first of the harmonic balance method, only the first two terms of the series expansion of the approximate periods (Eqs. (23) and (A30)) coincide with those of the exact period of the pendulum (Eq. (6)). This is due to the fact that for $N=1$ the exact differential equation of the pendulum and the approximated differential equation, where $\sin \theta$ has been substituted by Eq. (21), coincide only for small angles. In order to check the validity of the second approximation of the harmonic balance method in this case, it should be necessary to compare the approximate period of Eq. (A29) with the exact period of the linear differential equation:

$$
\frac{\mathrm{d}^{2} \theta}{\mathrm{d} t^{2}}+\omega_{0}^{2}\left(\theta-\frac{1}{6} \theta^{3}\right)=0
$$

which corresponds to a potential energy of the form:

$$
U(\theta)=m \omega_{0}^{2} l^{2}\left(\frac{1}{2} \theta^{2}-\frac{1}{24} \theta^{4}\right)
$$

It is possible to exactly solve the differential equation (A31) by using a similar procedure to that used to solve the exact differential equation of the simple pendulum for large amplitudes ${ }^{10}$. In this way it can be calculated the exact period for the oscillating system subject to the potential energy of Eq. (A32). Integrating Eq. (A31) and using the initial conditions in Eq. (2), it is possible to obtain the exact period of the oscillations as

$$
T_{\mathrm{ex}}=T_{0} \frac{2}{\pi} \sqrt{\frac{12}{12-\theta_{0}^{2}}} K(q)
$$

where $K(q)$ is the complete elliptical integral of the first kind and $q$ is given by the expression: 


$$
q=\frac{\theta_{0}^{2}}{12-\theta_{0}^{2}}
$$

If we expand in power-series this exact period (Eq. (A33)) we obtain the following expression:

$$
T_{\mathrm{ex}} \approx T_{0}\left(1+\frac{1}{16} \theta_{0}^{2}+\frac{19}{3072} \theta_{0}^{4}+\frac{420}{589824} \theta_{0}^{6}+\frac{10115}{113246208} \theta_{0}^{8}+\ldots\right)
$$

It can be observed that the three first terms of Eqs. (A30) and (A33) coincide, whereas the difference in the fourth term is $0.23 \%$ and that of the fith term is $0.72 \%$. 


\section{References}

1. Marion J B 1970 Classical Dynamics of Particles and Systems (Harcourt Brace Jovanovich, San Diego)

2. Fulcher L P and Davis B F 1976 Theoretical and experimental study of the motion of the simple pendulum Am. J. Phys. 44, 51-5

3. Ganley W P 1985 Simple pendulum approximation Am. J. Phys. 53 73-6

4. Cadwell L H and Boyco E R 1991 Linearization of the simple pendulum Am. J. Phys. 59 979-81

5. Molina M I 1997 Simple linearizations of the simple pendulum for any amplitude Phys. Teach. 35 489-90

6. Kidd R B and Fogg S L 2002 A simple formula for the large-angle pendulum period Phys. Teach. 40 81-3

7. Millet L E 2003 The large-angle pendulum period Phys. Teach. 41 162-3

8. Parwani R R 2004 An approximate expression for the large angle period of a simple pendulum Eur. J. Phys. 25 37-49

9. Hite G E 2005 Approximations for the period of a simple pendulum Phys. Teach. 43 290-2

10. Mickens R E 1996 Oscillations in Planar Dynamics Systems (World Scientific, Singapur)

11. Lim C W and Wu B S 2003 A new analytical approach to the Duffingharmonic oscillator Phys. Lett. A 311 365-73

12. Wu B S, Lim C W and Ma Y F 2003 Analytical approximation to largeamplitude ocillation of a non-linear conservative system Int. J. Non-Linear Mech. 38 1037-43 


\section{Figure captions}

Figure 1.- Relative error as a function of the amplitude for four different approximations. 


\section{Table 1}

\begin{tabular}{ccccccc}
\hline$\theta_{0}$ & $T_{\text {ex }} / T_{0}$ & $T_{\text {app } 1} / T_{0}$ & $T_{\text {app2 }} / T_{0}$ & $T_{\text {app3 }} / T_{0}$ & $T_{\text {app } 4} / T_{0}$ & $T_{\text {app }} / T_{0}$ \\
\hline $10^{\circ}$ & Eq. (4) & Eq. (22) & Eq. (25) & Eq. (28) & Eq. (31) & Eq. (34) \\
$20^{\circ}$ & 1.00191 & 1.00191 & 1.00191 & 1.00191 & 1.00191 & 1.00191 \\
$30^{\circ}$ & 1.01741 & 1.01759 & 1.01738 & 1.01738 & 1.01738 & 1.01738 \\
$40^{\circ}$ & 1.03134 & 1.03193 & 1.03125 & 1.03126 & 1.03126 & 1.03126 \\
$50^{\circ}$ & 1.04978 & 1.05129 & 1.04954 & 1.04957 & 1.04957 & 1.04957 \\
$60^{\circ}$ & 1.07318 & 1.07650 & 1.07261 & 1.07270 & 1.07270 & 1.07270 \\
$70^{\circ}$ & 1.10214 & 1.10877 & 1.10095 & 1.10119 & 1.10118 & 1.10118 \\
$80^{\circ}$ & 1.13749 & 1.14988 & 1.13512 & 1.13571 & 1.13569 & 1.13569 \\
$90^{\circ}$ & 1.18034 & 1.20249 & 1.17583 & 1.17716 & 1.17712 & 1.17712 \\
$100^{\circ}$ & 1.23223 & 1.27079 & 1.22393 & 1.22675 & 1.22664 & 1.22664 \\
$110^{\circ}$ & 1.29534 & 1.36175 & 1.28034 & 1.28608 & 1.28582 & 1.28583 \\
$120^{\circ}$ & 1.37288 & 1.48792 & 1.34607 & 1.35738 & 1.35675 & 1.35678 \\
\hline
\end{tabular}


Figure 1

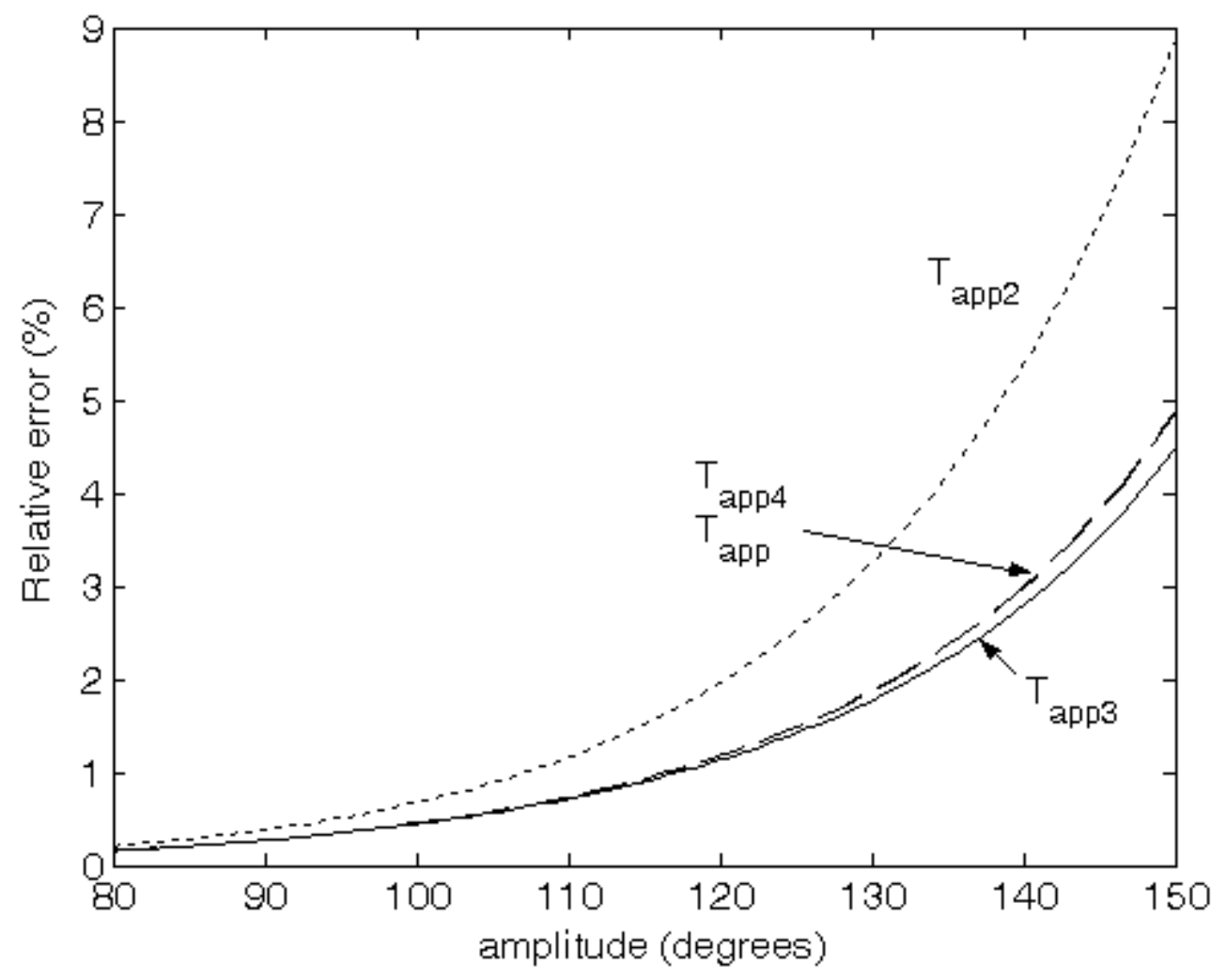

